European Journal of Sustainable Development

Research, 2018, 2(1), 11

ISSN: $2542-4742$

\title{
Environmental Engineering and Sustainable Development
}

\author{
Said Salah Eldin Elnashaie ${ }^{1^{*}}$ \\ ${ }^{1}$ University of British Columbia (UBC), Vancouver, CANADA \\ *Corresponding Author: nashaie@gmail.com \\ Citation: Elnashaie, S. S. E. (2018). Environmental Engineering and Sustainable Development. European \\ Journal of Sustainable Development Research, 2(1), 11. https://doi.org/10.20897/ejosdr/75554
}

Published: January 30, 2018

\begin{abstract}
Chemical Engineering is a very rich discipline and it is best classified using System Theory (ST) and utilized using the Integrated System Approach (ISA). Environmental Engineering (EE) is a subsystem of Chemical Engineering and also a subsystem of Sustainable Development (SD). In this paper both EE and SD are discussed from a Chemical Engineering point of view utilizing ST and ISA.
\end{abstract}

Keywords: sustainable development, environmental engineering, biofuels, bioproducts, sustainable development engineering

\section{INTRODUCTION}

Chemical engineering as a very rich discipline by its nature has witnessed considerable expansion in the last three decades which is consistent with the Amundson report (Amundson, 1988; Elnashaie, et.al., 1993). The most successful departments in the USA and Canada as well as most of the rest of the world have been those that expanded in the direction of becoming Chemical and Biological Engineering (CBE) Departments;(e.g.: University of Colorado, Boulder, Colorado, USA; University of British Columbia, Vancouver, Canada; Iowa State University, Ames, Iowa, USA; University of Alabama, Tuscaloosa, Alabama, USA, Department of Chemical and Biological Engineering, Rensselaer Polytechnic Institute, Troy, New York, USA ; Department of Chemical and Biological Engineering, Tufts University, Medford, MA, USA, etc.) with biological engineering meaning biochemical and biomedical engineering. Very similar names are, of course, also included in this category (e.g.: Division of Chemical and Biomolecular Engineering, School of Chemical and Biomedical Engineering, Nanyang Technological University, Singapore; Chemical and Biomolecular Engineering Department, University of Houston, Houston, Texas, USA; Chemical and Biomolecular Engineering Department, University of Notre Dame, Notre Dame, IN, USA, etc.). Other departments went half the way and became departments of Chemical and Bio-Chemical Engineering only (e.g.: Department of Chemical and Biochemical Engineering, Rutgers University, Piscataway, NJ,USA; Department of Chemical and Biochemical Engineering, University of Maryland Baltimore County, Baltimore, MD,USA; Department of Chemical and Biochemical Engineering, The University of Western Ontario, London, Ontario, Canada, Chemical and Biochemical Engineering Department, Instituto Tecnolo' gico de Veracruz, Veracruz, Ver., Mexico; etc.) and all these departments did reasonably well. Some Chemical and Biochemical Engineering Departments split into two separate departments (e.g.: University College, London University, London, UK) and both are doing reasonably well. Other departments became departments of Chemical and Materials Engineering (CME) (e.g.: Department of Chemical Engineering and Materials Science, Michigan State University, East Lansing, Michigan, USA, etc) and others became Chemical and Environmental Engineering (CEE) (e.g. The Department of Chemical and Environmental Engineering, University of Arizona, Tucson, AZ, USA, etc.) and did also reasonably well. It will be a great success to have all 4 disciplines (Chemical, Biological, 


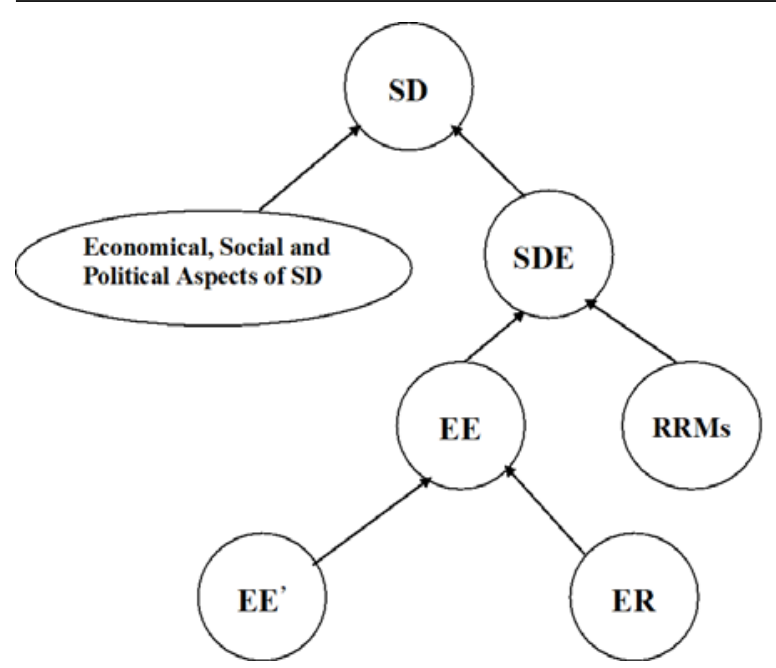

Figure 1. Simplified Diagram for SD and its Subsystems based on ST

Materials and Environmental Engineering) in one department and also to include the new comers discussed later and using a new approach suitable for such a Cross-Disciplinary $(\mathbf{C D})$ rich new specializations.

The 4 components are all Engineering Disciplines (EDs) and using the new approach will make them fall well under the same umbrella. However the $\mathbf{C D}$ approach extends to include not only EDs but also scientific disciplines such as chemistry, physics, mathematics, microbiology, etc. as well other disciplines such as economics, politics, sociology, etc. This in addition to the new comers discussed below will make the graduates truly ready for $\mathbf{C D}$ jobs in industry as well as for research work in both academia and industry as well as governmental research centers. It is also important to emphasis the fact that the new engineering era requires that graduates be prepared not only for design and operation, but also for innovation and should not be taught to learn only while in school and university but also to be taught the culture, ideology and philosophy of $\mathbf{L}$ ong Life Learning (L3 according to the ABET terminology). With all this branching, interaction and synergy between disciplines and these new requirements of modern industry and the healthy forms of globalization a new approach based on $\mathbf{S T}$ and may be called ISA should be adopted both in under-graduate and post-graduate teaching and research in academia and will naturally spread to industry and governmental research centers.

The 4 disciplines mentioned above are relatively well defined and discussed in the literature and the reader can be referred to excellent references for this purpose (e.g.: Wei, 1988; Amundson, 1988; Elnashaie et al., 1993; Vlachos et al., 1991; etc.). This does not mean that the last word has been said with regard to these disciplines, but it means that they are matured enough to be considered as given in a paper like this introducing some, may be, new and useful principles to this continuously expanding and complicating field. The first question now is: who are the new comers, and are they really new comers or intelligent extension of existing disciplines? These relatively new comers are: Sustainable Development Engineering, (SDE) which is a subsystem of Sustainable Development (SD) as a whole system (Elnashaie, 2008). In terms of ST and the ISA, we can consider SD itself as a system formed of technical and non-technical subsystems and SDE is a subsystem of the technical subsystem as shown in some details later. This relatively new comer is an extension of Environmental Engineering (EE) which is itself an extension of Efficient Engineering (EE' the dash is to distinguish it from EE). In other words if SDE (which is a subsystem of the technical subsystem of $\mathbf{S D}$ ) is a system then $\mathbf{E E}$ is a subsystem of it and $\mathbf{E E}$ is a subsystem of EE'. While in the terms of stability theorem $\mathbf{E E}$ ' can be considered necessary but not sufficient for $\mathbf{E E}$, what makes it sufficient is the introduction of Environmental Regulations(ERs) which came into the picture in the last 4 decades only in some countries while other countries did not introduce it in a proper manner affecting negatively the rest of the world. Similarly EE is necessary but not sufficient for SDE (which is itself necessary but not sufficient for $\mathbf{S D}$ ) what makes it sufficient is the introduction of Renewable $\mathbf{R a w}$ Materials (RRMs) to replace the Non-RRMs. This brings into the picture not only Bio-Fuels $(\mathbf{B F s})$ from wastes and energy crops (and not from food) and micro-algae, but also Integrated-Bio-Refineries (IBRs) since societies do not live only on fuels. This will be made clearer later. Figure 1 shows a diagram for $\mathbf{S D}$ and its subsystems.

Figure 2 shows a slightly more detailed diagram for $\mathbf{S D}$. 


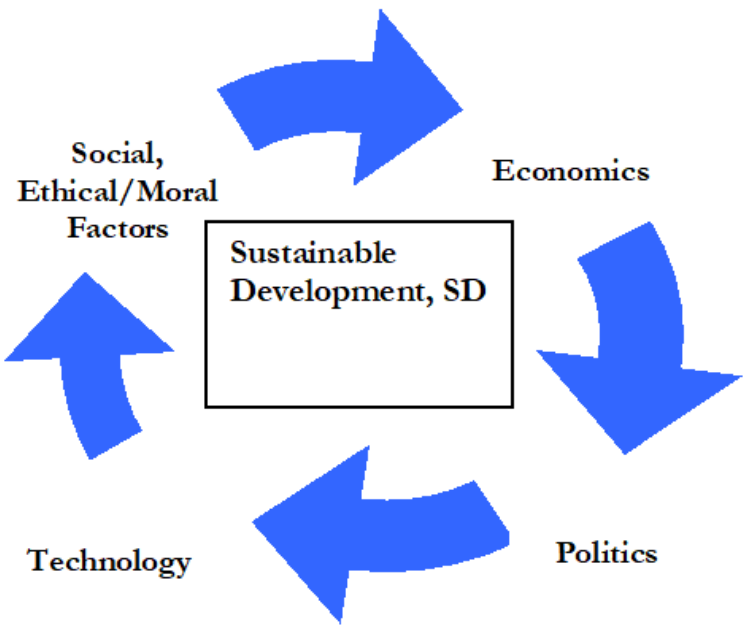

Figure 2. Another more detailed classification of SD

\section{Main Components of SD}

1- Political: e.g., legislations and strategic decisions....

2- Economical: e.g., investment in novel new technologies

3- Social, Ethical/Moral: e.g., consumption trends, acceptance of novel clean technologies and products, moral/ethical factors

4- Technological: e.g., novel efficient clean technologies, clean fuels, efficient utilization of renewable feedstocks, new environmentally friendly products, In-process Modification for Minimum Pollution Maximum Production (MPMP), efficient waste treatment.

Another relatively new and very important comer is Nano-Technology (NT), again using ISA, NT is an advanced part of materials science/engineering, but can NT develop without $\mathbf{C D}$ research including physicists, chemists, electrochemists, etc.? The answer is no and it is actually an advanced system formed of many subsystems and a discipline strongly cross-linked to many other disciplines.

Another relatively new comer is the practical implications of Non-Linear Dynamics (NLDs), Bifurcation (B) and $\mathbf{C h a o s}(\mathbf{C})$. These are actually very old disciplines, some philosophers consider it as old as the great French mathematician Poincare (e.g.: Smith, 1974; Stewart, 1989; Hawking, 1989) or even older while other philosophers consider it as old as the great Persian poet Omar-El-Khiam (Elnashaie and Elshishini, 1996), both are right in a way. What is really new and challenging is the practical implications of NLD, B and C especially in CBE systems, not because these are the richest systems in NLD, $\mathbf{B}$ and $\mathbf{C}$ but because they are the systems that the author knows more about (e.g.: Iscol, 1970; Elnashaie and Elhenawi, 1979; Edwards and Kim; 1998; Elshishini and Elnashaie, 1990; Elnashaie and Garhyan, 2004a, b; Garhyan and Elnashaie, 2004a, b, c; Chen and Elnashaie, 2004a, b; Mahecha-Botero et al., 2004; Elnashaie et al., 2006) . Complexity (COMP) is also quite important and it does not mean only numerical/computational COMP, but other forms of COMP, e.g.: simple configurations having COMP behavior and COMP configurations having simple behavior, etc. (Elnashaie and Grace, 2007). It is also important to realize that COMP is strongly related to NLD, B and $\mathbf{C}$ and will be kept under this category. This paper will address a part of the tip of this iceberg, in an organized manner to be as useful as possible for other researchers to develop the ideas much further. The tools are from ISA based on ST. The objective is a systematic organized framework relating: CBE, ME (Materials Engineering) and $\mathbf{E E}$ together with the relatively new comers of: SDE, NT and practical implications of NLD, B and C.

\section{THE ST AND THE ISA}

1. It seems reasonable to start by $\mathbf{S T}$ and ISA since they are the main tools. ST is the basis of the ISA which is the most efficient methodology for classification, organization and transfer of knowledge (Smith, 1974; Stewart, 1989; Vlachos et al., 1991; Elnashaie and Garhyan, 2003; Elnashaie and Uhlig, 2007). The approach of ST is very valuable in both research and education. In research, it is one of the most important components for the development of new knowledge and novel processes, especially in areas where $\mathbf{C D}$ research and development is a must for innovative solutions. SD is one of those areas which are $\mathbf{C D}$ by their very nature as briefly discussed above and will be discussed in some more details later on. The theme of this part is not to stress only the importance of the $\mathbf{S T}$ when dealing with the issue of $\mathbf{S D}, \mathbf{B F}$ and $\mathbf{I B R}$ but also to use it in developing the ISA as a tool for investigating and discussing all issues mentioned above 
including the $\mathbf{C D}$ educational methodology. It is also related to Mathematical Modeling (MM) the main tool for Computer Simulation (CS) and the maximum exploitation of Digital Computers (DCs) in education, research, innovation and optimal operation and control. ST is shown to be the best approach to organize knowledge and transfer it in an organized and easy manner. By this we believe that $\mathbf{S T}$ is the basic tool for dealing not only with SD but also to be widely used in engineering education, research and industry in relation to society. A definition of some important terms in ST is essential to build on towards ISA.

\section{ST}

\section{What is a system?}

The word system derives from the Greek word "systema" and means an assemblage of objects united by some form of regular interaction or interdependence. The main difference between $\mathbf{S T}$ and Set Theory (ST" to distinguish it from ST) is that in ST there is synergy between the subsystems while in ST" we are dealing with "dead" sets formed of "dead" subsets where the set is simply the addition of the subsets with no synergy or in other words no new characteristics which do not belong to the subsets. This is not the case with regard to systems and subsystems. A simpler more pragmatic description regarding systems includes:

- A system is a whole composed of parts (subsystems or elements)

- The concepts of a system, subsystems and elements are relative and depend upon the degree of analysis. For example, we can take the entire human body as a system, while the heart, arms, liver, etc. are elements. Or we can consider any of these elements as the system and analyze it in terms of smaller elements (or subsystems) and so on. In the other direction we can consider a country as a system and its cities as subsystems and the people as subsystems of the subsystems and the parts of these humans as elements if the analysis stops at this level or subsystems if the analysis continues.

- The parts of the system can be parts in the physical sense or they can be processes. In the physical sense the parts of the human/animal body or of a chair form a system. On the other hand, for chemical equipment performing a certain function, we can consider the various processes inside (within the boundary) of the system as elements which are almost always interacting with each other in a complex manner to fulfill the functions of the system. A simple chemical engineering example is a chemical/biochemical reactor in which processes like reactions, mixing, heat evolution/absorption, heat transfer, mass transfer, etc. take place to achieve the goal of the reactor, which is to convert reactants/substrates to products. A system can be formed of both, i.e. different parts of the system (a reactor and a regenerator combined to form a Fluid Catalytic Cracking (FCC) unit), each part having a number of processes taking place within its boundaries and the different parts are interacting through catalyst circulation and in each vessel the different processes are interacting and all types of interaction are giving the overall characteristics of the FCC unit.

- The properties of the system are not the sum of the properties of its components (elements or subsystems), although they are, of course, affected by those components. Instead, the properties of the system result from non-linear interaction (synergy) between components (elements or subsystems). For example, humans have consciousness, which is not a property of any of its components (elements or subsystems) separated alone. Also, mass transfer with chemical reaction has certain properties, which are not properties of chemical reaction or mass transfer alone (for example, multiplicity of steady states, as discussed below). This shows the great difference between $\mathbf{S}$ T and $\mathbf{S T}^{\prime}$ as mentioned above. ST' does not show such synergy, the set of numbers can be divided into the odd numbers and the even numbers subsets, the addition of them gives the set of numbers without any synergy unlike the $\mathbf{S T}$ which relates more to physico-chemical systems and shows that one plus one is equal two only in arithmetic.

\section{The state of the system and state variables}

The term "state of the system", rigorously defined through the state variables of the system, is used extensively. These state variables are chosen according to the nature of the system and the utilization and/or manipulation of it.

The state of a boiler can be described by temperature and pressure, a heat exchanger by temperature, a nonisothermal reactor by the concentration of the different components and temperature, an isothermal absorption tower by the concentration of different components on different plates, a human body by blood pressure and temperature, flow through a pipe by the axial and lateral profiles of local velocity and pressure. Thus state variables are variables that describe the state of the system.

\section{Input variables (parameters)}

They are not state variables. Instead, they are external to the system but affect the system, i.e. "work on the system". For example, the feed temperature and composition of the feed stream to a distillation tower or a chemical reactor or the feed temperature to a heat exchanger are input variables.

They affect the state of the system, but are not affected by the state of the system (except when there is a feedback control or a recycle, in which case we distinguish between control variables and disturbances or input 
variables). Of course, this again depends upon the level of analysis, or the boundary of our system (subsystem), for in many cases these input variables to our system are outputs (or state variables) of a previous system (subsystem).

\section{Design variables (parameters):}

They are associated with the design of the system and are usually fixed. Examples are the diameter and height of a Continuous Stirred Tank Reactor (CSTR) or of a tubular reactor.

\section{Physico-chemical variables (parameters):}

They are the physical and chemical parameters of the system, e.g.: viscosity, mass and heat transfer coefficients, equilibrium constants, reaction rate constants, etc.

\section{Boundaries of System}

As encountered above, a system has boundaries distinguishing it from the surroundings or environment. The relation between the system and its environment leads to one of the most important and fundamental classifications of systems:

1. Isolated System: It does not exchange matter or energy with the surroundings. It tends to the state of thermodynamic equilibrium (maximum entropy). An example is a batch adiabatic reactor.

2. Closed System: It does not exchange matter with the surroundings, but it does exchange energy. Such a system, again, tends to thermodynamic equilibrium (maximum entropy). Batch non-adiabatic reactors are typical examples.

3. Open System: It exchanges matter and energy with the surroundings. (notice that exchange of matter automatically implies exchange of energy). It does not tend to thermodynamic equilibrium but to steady state or what should better be called a "stationary non-equilibrium state", characterized by minimum entropy generation. A CSTR is a lumped example (state variables are not changing with the space dimension(s)), whereas a Tubular Continuous Flow Reactor (TCFR) is a distributed example (state variable are changing with the space dimension(s)). It is important to notice that open systems belong to the category of dissipative systems discussed below.

Flow systems are usually open, with one or more fluid streams entering and discharging from the boundaries. However, mixing vessels and flow loops can be closed systems, with energy transferred across their boundaries (e.g. by impellers of pumps) to provide the energy needed to initiate and maintain the motion, most or all of this energy ultimately being dissipated due to viscosity.

The above shows that the term "steady state" commonly used in CBE is not really very accurate, or at least it is not precise enough. A more accurate phrase should be "stationary non-equilibrium state", which is a characteristic of open systems, distinguishing it from "stationary equilibrium state", associated with isolated and closed systems.

\section{Steady, unsteady states and thermodynamic equilibrium of systems}

As briefly stated above, the steady state (stationary non-equilibrium state) is a concept related to open systems (all continuous CBE processes are open systems). Steady state occurs when the state of the system stops changing with time, but the system is not at thermodynamic equilibrium, i.e. the processes inside the system do not stop and the stationary behavior with time is due to a balance between input, output and processes taking place within the boundaries of the system. This steady state of lumped systems is a point in a space having the same dimensions as the problem (number of components + temperature + pressure, etc.), whereas that for distributed systems are profiles in the space co-ordinate(s) as additional dimension(s). Unsteady state of an open system starts at an initial condition and tends with time towards a steady state when the system is stable (a point for lumped system and profile for distributed systems). This initial condition for lumped systems is a point in the space dimension of the problem, while for distributed systems it is a profile in the space co-ordinate(s) as additional dimension(s). What if the system is unstable? This is addressed in a later section about NLD, B, C and COMP. On the other hand, for isolated and closed systems the unsteady state changes with time, tending towards thermodynamic equilibrium, which is stationary with time because all processes have stopped (the process is "dead").

For flowing systems (leaving aside ideal, i.e. in viscid, fluids and fluid static), energy is required to actuate fluid motion and maintain steady flow and transport processes. If the source of energy (e.g. a pump, potential energy, or a heat source) is terminated or exhausted, then the system asymptotically approaches the thermodynamic equilibrium state of a static isothermal system.

\section{ISA}

The current lack of success in improving industrial sustainability, coupled with the challenges of bio-complexity and resilience, indicates that sustainability is a system's problem requiring collaborative solutions with $\mathbf{C D}$ nature. Other issues introduced in the introduction follow the same route. A number of technical advances will likely improve the usefulness of models, including rigorous methodologies for dealing with missing and uncertain 
information; improved methods for interpretation of multivariate data sets and for multi-objective decision making involving trade-offs among conflicting goals; and novel modeling methods as alternatives to traditional MM. More generally, there is a great need for operational definitions and metrics for sustainability and resilience in economic, ecological, and societal systems. SD in a changing global environment will require resilience at many levels, including human communities and economic enterprises. In the face of ever-increasing global COMP and volatility, it is essential to move beyond a simplistic "steady state" model of sustainability. Instead, we need to develop adaptive policies and strategies that enable societal and industrial institutions to cope with unexpected challenges, balancing their need to be able to achieve efficient sustainable $\mathbf{B F}$ s as one of the many challenges imposed by modern industry and societies and reflecting themselves upon education, industry, research and the entire society in a global manner. ISA is the use of ST to tackle all these challenges in an integrated, efficient and well organized manner. ISA utilizes $\mathbf{M M}$ and $\mathbf{C S}$ to the highest level coupled to experimental work and organized exploitation of industrial data not only to improve operation but to discover novel processes. It maximizes utilization of present technologies using on-line efficient computer optimization and control with objective functions being not only Maximum Productivity but also Minimum Pollution (MPMP). It does not stop at that; it also considers conceptual optimization which may change the technology fundamentally, i.e.: from fixed bed to fluidized bed by Grace and co-workers in UBC (e.g.: Abo-Ghander et al., 2012 ; VIgneault et al., 2012; Grace, 2015; etc.) and Elnashaie and co-workers in Auburn University and UBC (e.g., Elnashaie and Elshishini, 1996; Chen and Elnashaie, 2004; Elnashaie and Uhlig, 2007; Elnashaie, 2008; etc.) and many others; using membranes to "break" thermodynamic equilibrium barriers for reversible reactions and integrated membrane reactors having complementary reactions at both sides of a suitable membrane by Elnashaie and others as well as combining endothermic and exothermic reactions to achieve an auto-thermal process by Elnashaie and others; a continuous reactor-regenerator process such as Fluid Catalytic Cracking (FCC) and its continuous development by petroleum companies, Pressure Swing Adsorption (PSA) to replace absorption-regeneration, Pinch Technology (PT) for maximum energy efficiency in Heat Exchangers (HEs) Net Works (NWs) and its extension to Mass Transfer (MT) NWs by El-Halwagi and co-workers at UCLA, Auburn and Texas A\&M (e.g.: El-Halwagi, 2006), novel polymerization processes such as the fluidized bed UNIPOL process for polyethylene and polypropylene and their further development and also the bifurcation and chaotic characteristics of these and other polymerization reactors by Ray, Teymour and others at Wisconsin, Madison and Illinois Institute of Technology (IIT) (e.g.: Teymour, 1989; Teymour and Ray, 1992a,b; Ray and Villa, 2000). The list is endless and efforts toward novel designs using conceptual de-bottle-necking are intensifying in academic research, research centers and industry.

\section{ADVANCED CD ENGINEERING EDUCATION}

Now after briefly introducing the ST and ISA concepts we move to Engineering Education, not only with regard to $\mathbf{C B E}$ education and its branching but also with respect to Engineering Education as a whole with special emphasis on CBE and its CD branching. (NCSE, 2003 and NGS-NANO, 2008). In the last two decades Engineering has been quickly entering a new phase. The speed of transformation has been accelerating and the boundaries between different engineering disciplines are falling down very fast (Klein, 1990; Lattuca, 2001; Lattuca 2004; JEE, 2005, 2006; Borrego and Newswander, 2008). In addition, the boundaries between engineering, with its different specializations, and other scientific disciplines (chemistry, microbiology, mathematics, etc) are becoming more permeable. Not only that but also the engineering disciplines is becoming more integrated to socioeconomical, political and moral/ethical issues. In addition the world economy is moving from national economies with continuously intensifying exchange to a global economy which is much more integrated and competition is more intensive. More over, awareness of the danger of depleting the non-renewable resources and exceeding the absorption capacity of our ecosystem is increasing. This state of affairs is calling for a radically new approach not only with regard to engineering research but also EE. Engineering is branching into more specializations, while at the same time is calling for closer integration between different engineering, scientific and humanitarian specializations. In addition to the four main disciplines (CBE, Mechanical Engineering (ME) and EE), among the new comers SD gave birth to SDE and even before fitting the other newcomers (NT, NLD, B and C) into the framework the situation becomes quite complex. SDE shows very clearly that $\mathbf{E E}$ ' and $\mathbf{E E}$ are necessary but not sufficient for sustainability. We can have very efficient designs and very clean technologies but they are not sustainable because they do not use RRMs. Sustainability is becoming the modern focus of all research and development and the main challenge facing science and technology in the twenty first century. Research on renewable energy is very important and is intensifying both in academia and industry. This is good, but modern societies do not live only on energy, there are other products and commodities that have to be produced from RRMs. Here comes strongly into the picture the concept of IBRs which will not only produce clean renewable BFs but also other products and commodities. 
When we add up the above 4 basics and 3 newcomers we have a huge engineering building attached to all other engineering and scientific disciplines. This huge CD engineering complex has its foundations based on fundamental knowledge; technological progress is limited by these foundations. Therefore fundamental research is extremely important in this challenging and interesting episode for the good of the entire world. The link between scientific discoveries and technological innovation through transformational engineered ISA is becoming very important.

These challenges call for a $\mathbf{C D}$ research involving all types of the continuously evolving human knowledge. This CD research is evolving continuously and will create new ideas and innovative breakthroughs not expected before. This challenging and promising new trajectory needs a tool to help organize it and bring it to useful and dynamic conclusions. The ISA based on ST both discussed above are the best tools for the generation, organization and transfer of knowledge with positive synergetic effects maximizing the return on investment for research and development.

There are strong evidence that there is some contradiction between sustainability and profitability some researchers are even claiming that there is a contradiction between sustainability and the second law of thermodynamics. The most suitable solutions to these contradictions are through innovation which is, from an informatics point of view, a very good source of negative entropy. From the above brief introduction we can clearly point to the following general needs of modern advanced economies and societies as briefly given below:

1. Develop a culture of Discovery and Innovation $(\mathbf{D \& I})$ through the entire education/research system with special emphasis on engineering education and research.

2. Find Innovative Solutions (ISs) for the contradiction between sustainability and profitability.

3. Develop an efficient approach for linking fundamental scientific discoveries to engineering applications, Efficient Science to Technology (EST). The best such approach is an integrated $\mathbf{C D}$ approach based on ST.

4. Develop among the different generations of engineers a strong spirit of entrepreneurship and the development of high-caliber engineers with strong CD capabilities suitable for leadership in a global economy. This includes developing among undergraduate and graduate students the creative process and cross-cultural collaboration. The pre-college education including institutes, teachers and students is also an integral part of this process.

5. Expanding the use/development of $\mathbf{M M}, \mathbf{C S}$ computer and optimization techniques.

6. Expand the domain of $\mathbf{E E}$ which aims at clean technologies to $\mathbf{S D E}$ aiming at $\mathbf{S D}$.

7. Develop an engineering awareness of the technological implications of global economy in successfully explore and realize innovation opportunities and thus qualified to be in the forefront of competition in a global economy.

8. Develop strong relations between, academia; small innovative firms; relevant large agricultural and industrial producers as well as federal, state and local authorities. These relations should be aiming at intensifying discoveries, innovation and their commercialization nationally and internationally. This will include not only CD research and development but also education and continuous training.

9. In a global economy, teaching, research, design and production cross national borders calling for the development of partnership with foreign universities, research centers and industries for teaching, training, research co-operation and innovation in a global economy. Develop different categories of $\mathbf{C D}$ engineering departments each one involving an optimally chosen number of disciplines in order to achieve an integrated CD operation. These departments should be formed of diverse and talented domestic and international individuals who will prepare diverse talented individuals who can function in global economy, where design, production and innovation efforts cross national borders.

Different engineering departments are needed with different engineering disciplines in order to achieve a good step forward in the arena of strong competition in a growing global economy.

The framework of the above 10 general characteristics (summarized in Figure 3) is essential for starting a successful CBE department including the 4 well established disciplines in the right way and also encompassing the 3 main new comers. The department will have the components of the typical present $\mathbf{C B E}$ departments classified on the basis of ISA as follows in addition to necessary fundamental subjects such as chemistry, biochemistry, mathematics, physics, computer programming and humanities. This will mainly include:

1. Material and energy balances for chemical and biochemical reacting and non-reacting systems.

2. Transport phenomena

3. Mass Transfer

4. Heat transfer

5. Sizing of reacting and non-reacting chemical and biochemical systems

6. Process control for reacting and non-reacting chemical and biochemical systems 


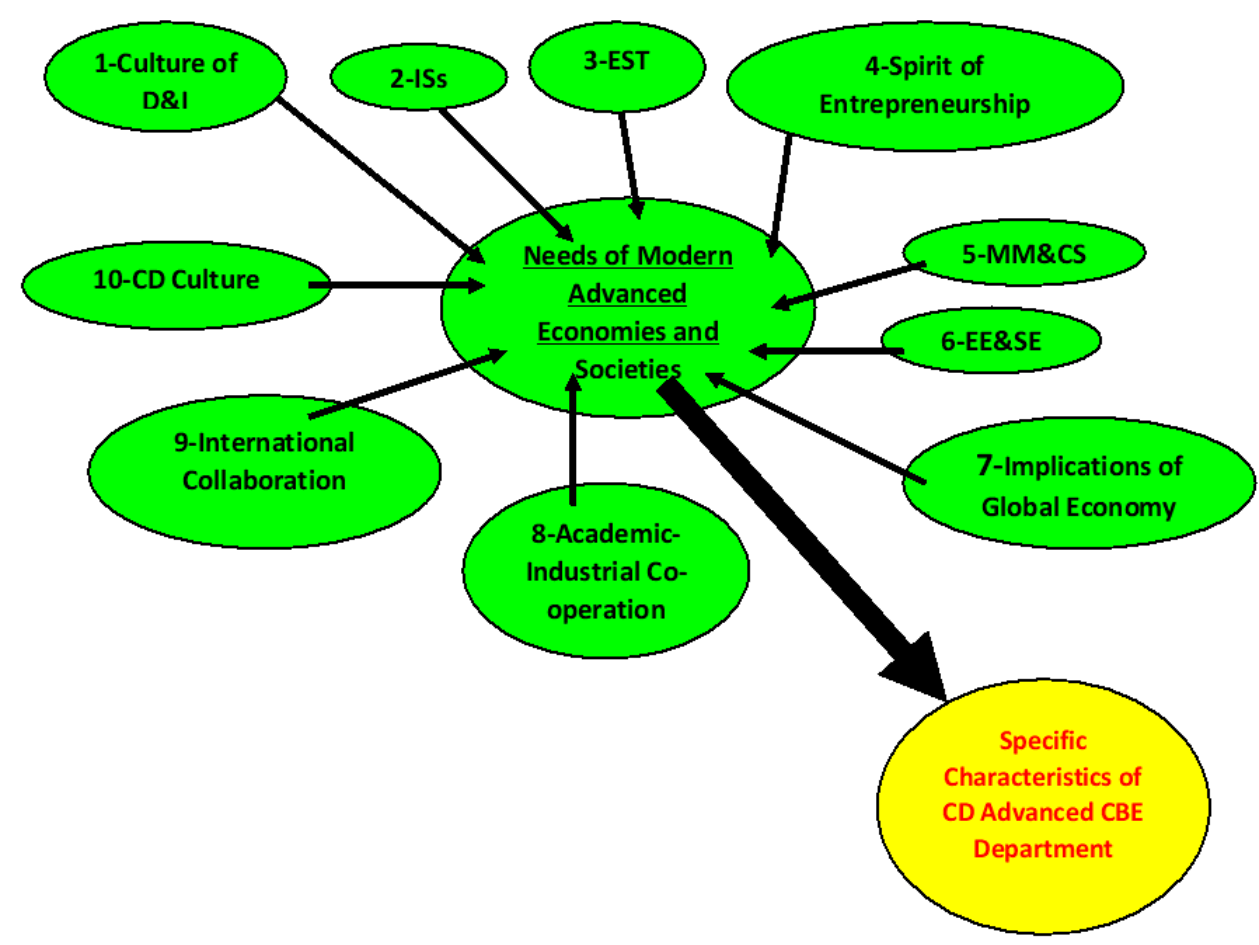

Figure 3. 10 needs of Advanced Economies and Societies and output to a CBE department

7. Momentum balance; momentum transfer and fluid dynamics

8. Plant design and economics

In addition to the classical components, condensed and organized using an ISA, such a department will have special emphasis regarding research and education on the points shown below and condensed in Figure 4 with the outcome of Figure 3 (the green box in Figure 3) as an umbrella:

1. BFs: including developing and optimization of novel efficient compact processes and comparing them as renewable energy sources. A good preliminary plan is to concentrate on:

- Cellulosic Bio-ethanol using different feed-stocks and different processes, e.g.: Lignocelluloses fractionation followed by hydrolysis of cellulose/hemicelluloses; followed by fermentation. The emphasis should also include bio-butanol because of its advantages over bioethanol. Novel processes, novel modes of operation as well as novel mutated microorganisms will be combined to exploit the positive synergies between these factors to achieve highest possible efficiency. Continuous Stirred Tank Fermenters (CSTFs) as well as packed bed immobilized fermenters are to be considered. Different hydrolysis techniques should also be considered, both acidic and enzymatic, to reach the most efficient route for sugars production from cellulose/hemicelluloses. In the fermentation step, different techniques for continuous ethanol removal are to be considered to maximize breaking the inhibitory effect of ethanol on the fermentation process. Unusual modes of operation (Chaotic fermenter (Elnashaie and Garhyan, 2004a)) are also to be explored. Novel mutated microorganisms are to be developed in collaboration with microbiologists from other departments to ferment all the types of sugars resulting from the hydrolysis step, and to be tested in classical and novel membrane fermenters.

- Bio-hydrogen, including producing it through biological processes from non-bio raw materials (e.g.: biological Water Gas Shift (WGS) of CO) and also thermo-catalytic from bio raw materials (e.g.: hydrogen extraction from syngas produced from biomass through gasification or catalytic gasification or fast pyrolysis of biomass to bio-oil followed by steam reforming of bio-oil), or bio-processing of biomass (biological treatment of well chosen biomass using suitable microorganisms). Both in-situ as well as exsitu removal of hydrogen will play an important role in the cut-edge development of these processes to make it competitive with the classical catalytic steam reforming of natural gas and its recent improvements using bubbling and circulating fluidized bed membrane reformers.

- Biodiesel, including the short term small scale bio-diesel from vegetable oils (or used oils) through transesterification using metals or enzyme catalysts and suitable alcohols. However the main thrust will be regarding the long term, large scale production of Fischer-Tropsch bio-diesel (FT-bio-diesel) from syngas produced from biomass through one of the processes described above. The research should compare the two routes for syngas production with special emphasis on the percentage of the valuable component, hydrogen, in the syngas. The research should concentrate on developing a novel suitable 


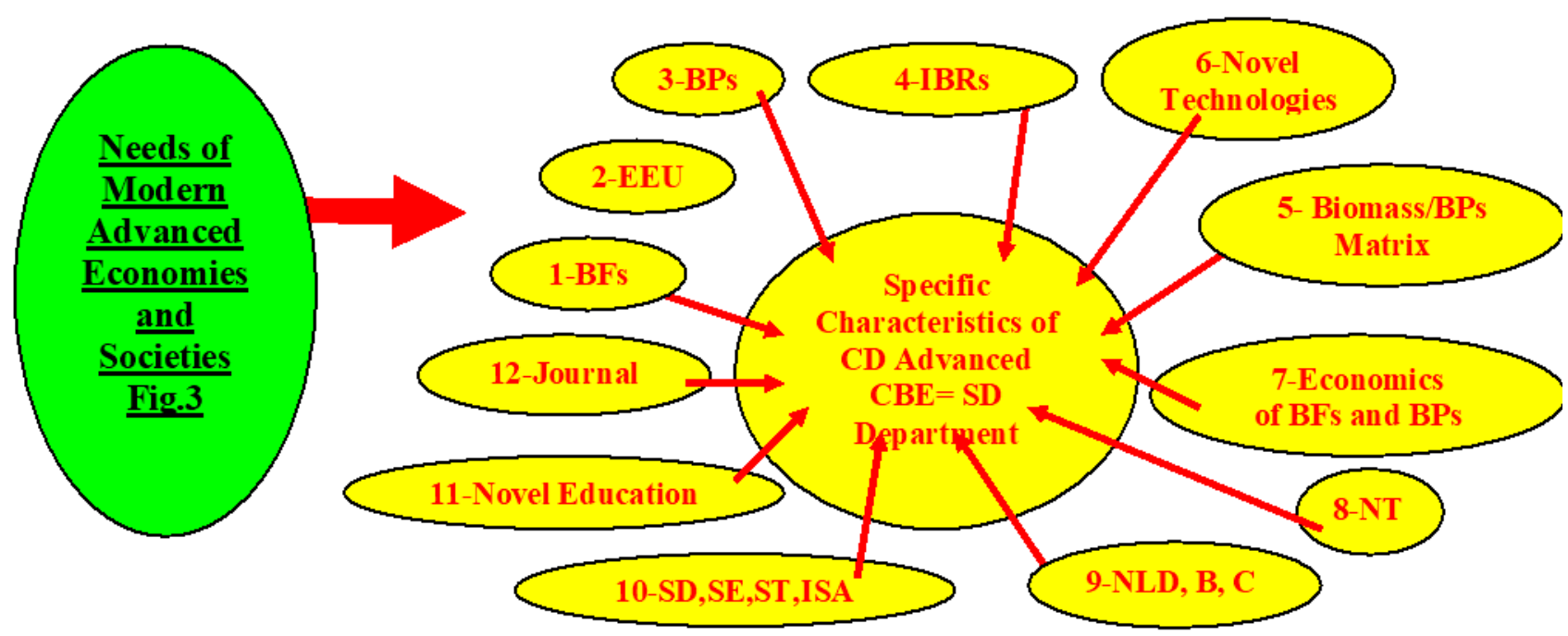

Figure 4. Needs for Figure 3

and efficient membrane Circulating Fluidized Bed (CFB) process for the steam reforming of bio-oil as well as a novel FT process that operates at relatively high temperatures and with a syngas having a very high yield of $\mathrm{H}_{2}$ and $\mathrm{CO}_{2} / \mathrm{CO}$ ratio which is a characteristic of the syngas produced from the efficient membrane reformer. Research will also extend to developing a novel compact auto-thermal membrane reactor combining both the endothermic steam reforming of bio-oil and the exothermic FT reaction of syngas to FT-biodiesel. The research will also include novel ideas to improve the fast pyrolysis and the catalytic gasification processes as well as the biomass collection, classification and pre-treatment before processing. In addition the different FT-bio-diesels produced will go through the standard tests, including diesel engines testing and these characteristics related to parameters associated with the raw materials and novel technologies used.

Such a department will also address in an objective scientific manner the dispute regarding Energy Ratio (ER) and Net Energy Ratio (NER) for bio-fuel and fossil fuels which will address the energy from quantitative as well qualitative points of view.

2. Efficient Energy Utilization (EEU): which includes many subjects related to both clean fuels (e.g.: hydrogen), BFs from RRMs, etc and will also include optimal clean utilization of fossil fuels. In this work it should be planned to improve the efficiency of the cleanest way to produce the clean fuel hydrogen, that is electrolysis and the cleanest and most efficient way to produce electric energy which is the fuel cells. The improvement will concentrate on biotechnology as the main focus by developing novel bio-electrolysis process and bio-fuel cells. Other techniques to improve the efficiency in the two processes and their interfaces should also be integrated with this biotechnology based improvements. Integrated electrolysisfuel cells are used in order to achieve continuous electricity source which is necessary for certain applications (e.g.: thermally efficient housing) from an intermittent clean source (e.g.: solar energy). The efficiency of this integrated process can be improved considerably using the suggested bio-electrolysis coupled to the biofuel cells. Research will also include the development of this integrated process in co-operation with the designers and developers of thermally efficient housing.

3. Bio-Products (BPs): they are very important part of SD due to the simple fact that, although most of the attention is going to the important subject of sustainable energy, societies do not live only on energy; there are many other commodities (consumer goods) and capital products that need to be replaced by sustainable BPs. Societies are alarmed about the fact that oil has passed its peak and will finish in a few decades, but what about other raw materials? In fact cupper is running short and its price is rocketing high. Thus research and preparation of engineers will not include only novel processes development, but also novel products development. The approach will depend upon the highlighting of the most critical raw materials that are dangerous to SD other than energy, as well as the commodities and capital products related to those raw materials coupled to the commodities and raw materials which are easiest to replace with replacements produced from RRMs. This essential classification and focusing process will be followed first by identifying the state of the available technologies related to these factors. This process will then lead to the most important novel processes and products to develop and to establish the research program to develop them efficiently and cleanly.

4. IBRs: it is a concept which is still at its infantry. Relatively recently NSF has offered a solicitation for the development of IBRs which will be the tool, in the near future, to achieve the above points in an integrated 
manner that is producing BFs and other BPs in one IBR. In order to have a clear vision of IBRs it is essential to realize what a $\mathrm{BP}$ (or BF) is? It is any product produced from a biological process even if the raw material is not a bio (e.g.: hydrogen from CO using biologically catalyzed WGS process). It is also any product produced from a bio-raw material using non-biological process (e.g.: biomass to bio-diesel using any technique for the production of syngas followed by FT process). It can also be both, i.e.: the raw material and the process are both bio (e.g.: Cellulosic waste to cellulosic bio-ethanol through a number of steps most of them are bio-processes). Beside biomass and other forms of wastes there are also special crops which are being developed to be used for BFs and other BPs (e.g.: switch grass). In addition there are very important bio-raw materials which are treated now as waste or cheap fuels such as lignin which is separated from different types of lingo-cellulosic wastes (especially agricultural waste such as rice straw). Through intensive innovative $C D$ research this lignin can be a base of a part of IBRs to compete, and eventually replace, the present petrochemical industry based on none-RRMs. Well directed research can also produce sugars for fermentation to ethanol from this lignin waste which will increase the yield of the cellulosic bio-ethanol process more than $30 \%$. It is also important to recognize the meaning of RRMs, this is very relative, for, theoretically speaking, fossil fuel is also renewable, but its cycle is a million years or more. So what we mean by renewable raw materials is a raw material which is renewable over few months up to a maximum of a couple of years. We should also add that its Life Cycle Analysis (LCA) should show a net CO2 (and any other harmful side product) of almost zero, i.e.CO2 produced is consumed in photosynthesis to produce almost the same amount of renewable raw materials. The socio-economical implications of IBRs will include both production and consumption sustainability. Research should address, in a CD integrated manner, all technological, socio-economical, political and moral/ethical aspects.

5. Biomass/BPs Matrix: Other efficient utilization of RRMs especially biomass, although the above bioenergy and other bio-products represent the core of biomass utilization for SD other utilizations are possible. Bioprocesses are very location sensitive and vary very widely from one place to the other and even with the change of circumstances in the same place. For example, in some places some kinds of biomass will optimally be used for the production of composites for the agriculture soil, in other places it will best be used as an addition to buildings materials, etc. Some animal residues also can have either general applications in production of bio-fuels and bio-products or special applications for special products or services. This part of the research and education should involve a classification of the different types of biomass, their geographical availability within the US, Canada or any other country around the world together with the different products possible from the different types of biomass. It should be a matrix of biomass RRMs as columns and possible products as rows with the technology suitable for each element of this matrix corresponding to a certain RRM and a corresponding product. It will be shown later that an IBR will occupy a certain part of the matrix or the entire matrix. It is also important to realize that each such matrix element does not correspond to a single technology relating the specific RRM to the specific product. Therefore the process will also include surveys of the existing technologies to produce these products from these categories of biomass and the needed novel technologies to achieve that when no technology is available or the available ones are not efficient or not environmentally friendly. Research and education will also address the non-technical factors associated with the choice of the optimal products and optimal processes from specific types of biomass with special emphasis on the characteristics of the locality of the specific biomass.

6. Development of Novel Technologies: this is to be achieved through different intellectual innovation processes, e.g.: sequential de-bottlenecking. The process of developing novel technologies is much more difficult than optimizing and improving the efficiency of an existing technology and both are important. This is due to the fact that in last few decades lots of very efficient, modeling and computer tools has been developed to efficiently achieve the optimization of existing technologies. However the development of novel technologies takes lots of intelligent, intellectual efforts and development on the basis of a culture of discovery and innovation which is not strong enough in our present educational system. Innovation includes both novel configuration (e.g.: efficient fluidized bed membrane steam reformer for hydrogen production instead of the inefficient present fixed bed reformer constrained by thermodynamic equilibrium limitations + intra catalyst particles diffusional limitations + heat transfer limitations) and novel modes of operations (e.g.: periodic or chaotic autonomous or non-autonomous processes leading to higher yields and productivity compared with optimal steady states). It may also involve a novel product or service. The suggested center will work hard to become a focal point not only for developing specific novel technologies but also to develop the culture of discovery and innovation and to develop all possible intellectual tools for this process, e.g.: sequential de-bottlenecking.

In addition, novel configurations usually have more complex configurations than classical configuration (e.g.: CFB membrane reformer compared with fixed bed reformers). This gives rise to mechanical and 
hydrodynamic challenges which will be tackled in the center both experimentally and using advanced computer modeling and simulation.

7. Economics of BFs and BPs: making these bio-processes and bio-products economically competitive, socially acceptable, sustainable and environmentally friendly as well as gaining political support for it. The main bottleneck for bio-fuels and bio-products is certainly economics. The main challenge is to break the contradiction between sustainability and profitability, in other words to present to the consumers products which are reasonably priced and for the producers (and the rest of the production-consumption chain) products to achieve reasonable profit for the economical cycle. However, and despite the major importance of economics, other social, political and moral/ethical factors are not negligible at all. All the factors contributes to the success of achieving sustainable economy which is also clean and leads to peaceful coexistence between peoples. The center will use a system approach in order to study the positive and negative synergy between these different factors associated with sustainable development and its relation to BFs and BPs.

8. Nano-Technology (NT): addressing this hot new comer in a CD manner and its applications in all fields of applications, especially chemical and biological engineering applications. This field opens new horizons in all applications and is multidisciplinary by it very nature. This new department will be the best focal points for the development of this strategic field in co-operation with other engineering and science departments.

9. NLD, B and C: this field (system) is usually considered to belong to the theoretical part of knowledge and investigation. However in the last three decades these phenomena are finding more practical applications and relevance both for autonomous systems as well as non-autonomous system. They are important to understand many phenomena which are not possible to understand them without having the fundamental knowledge associated with this field. All systems are actually non-linear and therefore there dynamics lend themselves to this field, linear system are actually approximations obtained by linearizing the system in the neighborhood of a specific state and therefore gives local characteristics but not global ones. Bifurcation has dismantled the boundaries between static and dynamic behavior and made them interdependent. This is a CD field by its very nature and is quite important to control when the behavior is harmful and exploit when it is useful. COMP belongs to this field and it is a very important subsystem of this system as briefly discussed above and shown later in more details.

10. SD, SDE, ST and ISA (Fiksel, 2006): sustainability is still young and many of its aspects are not completely clear even among academicians. A simple example is the question, phrased on $\mathbf{S T}$ terminology, is EE a subsystem of SDE or the opposite? The preliminary believe accommodated at this time by the research group of this proposal is yes it is a part of $\mathbf{S D E}$ and that $\mathbf{E E}$ is necessary but not sufficient for sustainability and that good efficient engineering is not sufficient for sustainability. This is due to the fact that you can have a very efficient, clean technology, but if it is based on non-renewable raw materials then it is not sustainable. However these concepts are still not deep enough, technologically, socio-economically, politically and from moral/ethical points of view. Even most definitions of sustainability and sustainable development are not very accurate and they are more catch-phrases than strong meaningful and survivable definitions. The suggested center will use system approach to move forward in the direction of establishing stronger, clearer and more meaningful definitions to these important concepts. This effort will help all involved as well and others to achieve all other tasks.

11. Novel Education: using CD teaching programs on the above subjects, preparing the graduates for leadership positions in a global economy as innovators. As we want to shorten the period between scientific discoveries and their innovative technological application through transformational engineered systems approach, we want also to achieve the same with respect to education. This is best achieved using a new approach for engineering education based on system theory. System theory is the best and most efficient mean to organize and transfer knowledge and create a common language between disciplines. This is due to its very nature and its definition of systems, subsystems, their boundaries and characteristics in general terms not restricted to specific system or disciplines. It is also the best mean to make the gap between scientific/technological discoveries and teaching/training as short as possible, which is very desirable in a very competitive global economy. The center will develop new educational programs based on system theory designed for:

a. strategically creating the capacity to initiate and exploit knowledge for technological innovation and minimize the gap between scientific/technological discoveries and education/training

b. maximize engineers ability to think positively and independently in contradistinction to memorizing or learning engineering as a craft

c. develop graduates experienced in the creative process and cross-cultural collaboration.

d. develop graduates able to define pathways to explore and realize innovation opportunities for success in a global economy. 
e. develop graduates who are leaders in a global economy

These educational programs will be formed of diverse (domestic and international) and talented individuals as teachers/trainers who are part of the research program of the center. They will prepare diverse talented individuals (domestic and international) who can function in global economy, where design and production efforts cross national borders. Diversity will also include cross-disciplinary team of faculty members and students that is diverse with respect to gender, race, ethnicity, disability, and culture.

12. New Journal: last but not least a suggested department for SD may pioneer the publication of a modern international SD engineering journal. The aim of this Journal is to be one of the tools to address the bioenergy, BFs, BPs, IBRs and sustainability challenges and make sure of publishing and spreading widely, nationally and internationally, the novel work of the department and similar departments worldwide, as well as relevant research nationally and internationally. The journal will emphasis novel ideas and make it reach the scientific community in an efficient manner. The Journal will aim at the publication and dissemination of original research, reviews and discussions on the latest developments in the fields of BFs, BPs, IBRs, sustainability and their synergic relations to each other and to global economy. It will also address challenging educational issues related to innovation and developing a new generation of innovative engineers suited for the global economy. It should also focus on research utilizing ST and ISA to develop novel technologies which contribute to SD for the entire planet and its entire population in the framework of global economy as well as advances on the foundations of fundamental knowledge. Papers may be theoretical (including computational), experimental or both. Also intellectual conceptual ideas to advance the culture of discovery and innovative thinking should be given special attention.

\section{CONCLUSIONS}

This paper uses System Theory to analyze the sustainable development and its relation to environmental engineering as well as sustainable development engineering. The analysis addresses both research and education giving suitable examples for both cases. It is clear that sustainable development and sustainable development engineering are more general than environmental engineering and are both more fundamental in both teaching and research. The presented approach will be very useful in education and research, complex disciplinary systems and useful for engineering specializations as well as other scientific and social disciplines.

\section{ABBREVIATIONS}

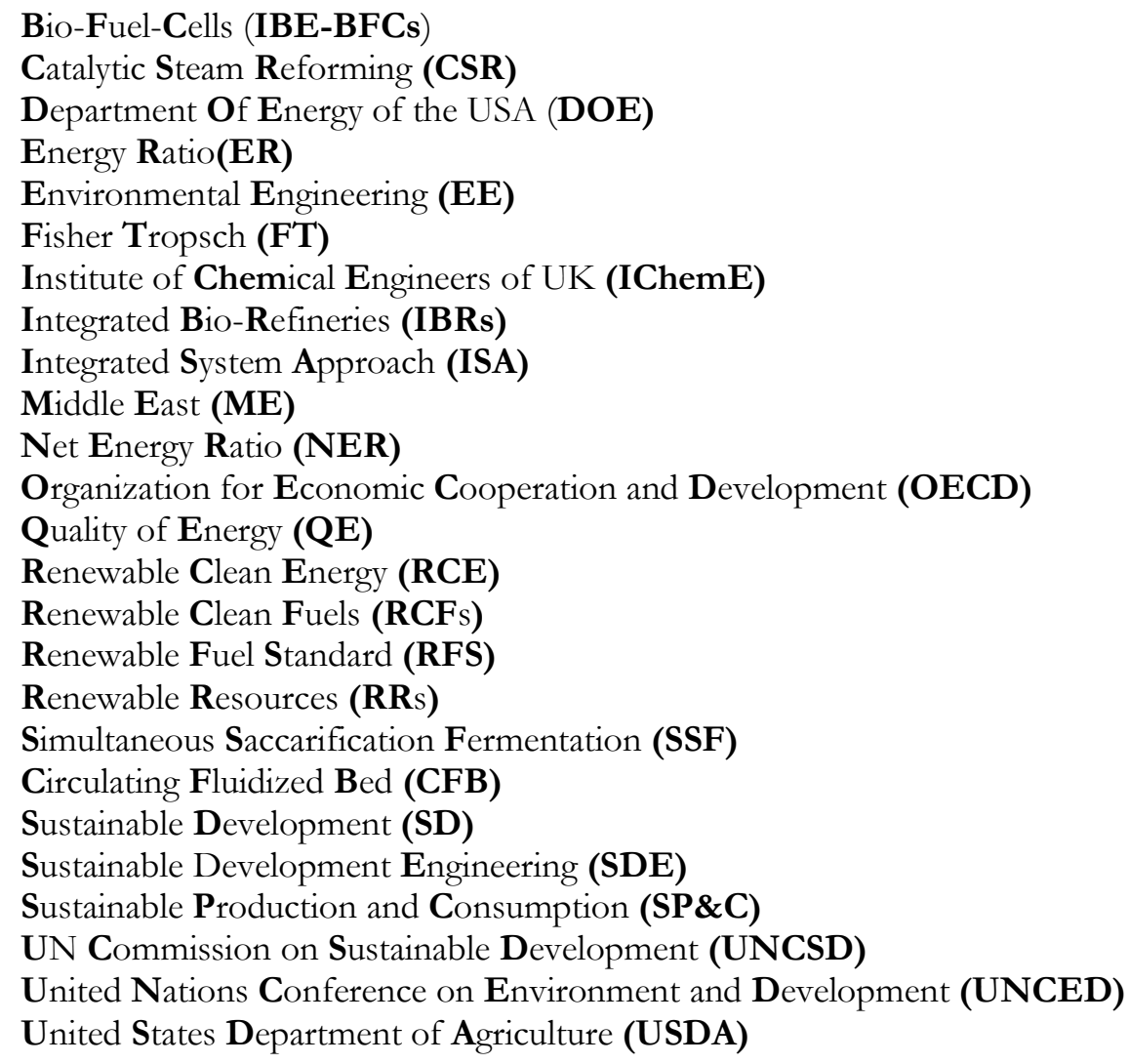




\section{REFERENCES}

Abo-Ghander, N., Logist, F., Grace, J. R., van Impe, J. F. M., Elnashaie, S. S. E. H. and Lim, C. J. (2012). Comparison of diffusion models in the modelling of a catalytic membrane fixed bed reactor coupling dehydrogen-ation of ethylbenzene with hydrogenation of nitrobenzene. Computers \& Chem. Engng, 38, 11-23. https://doi.org/10.1016/j.compchemeng.2011.10.007

Amundson, N. R. (1988). Frontiers in Chemical Engineering, Research needs and opportunities. National Academy Press, Washington, D. C.

Borrego, M. and Newswander, L. (2008). Characteristics of Successful Cross-disciplinary Engineering Education Collaborations. Journal of Engineering Education, 97(2), 1-23. https://doi.org/10.1002/j.21689830.2008.tb00962.x

Chen, Z. and Elnashaie, S. S. E. H. (2004a). Steady-State Modeling and Bifurcation Behavior of Circulating Fluidized Bed Membrane Reformer-Regenerator for the Production of Hydrogen for Fuel Cells from Heptane. Chemical Engineering Science, 59(18), 3965-3979. https://doi.org/10.1016/j.ces.2004.06.011

Chen, Z., Said, S. E. H. and Elnashaie, S. S. E. H. (2004b). Bifurcation Behavior and the Efficient Pure Hydrogen Production for Fuel Cells Using Novel Autothermic Membrane Circulating Fluidized Bed (CFB) Reformer. Industrial \& Engineering Chemistry Research, 43(18), 5449-5459. https:// doi.org/10.1021/ie0341799

Edwards, W. M. and Kim, H. N. (1998). Multiple steady states in FCC unit operations. Chem. Engng. Sci., 43, 18251830. https://doi.org/10.1016/0009-2509(88)87048-9

El-Halwagi and Mahmoud, M. (2006). Process Integration, Academic Press, USA.

Elnashaie, S. S. (2008). The Big Picture. The Chemical Engineer(tce), issue 803, 26-28.

Elnashaie, S. S. and Uhlig, F. (2007). Numerical Techniques for Chemical and Biological Engineers Using MATLAB, Springer, USA.

Elnashaie, S. S. E. H. and El-Hennawi, I. M. (1979). Multiplicity of the steady states in fluidized bed reactors, IV. Fluid catalytic cracking. Chem. Engng. Sci., 34, 1113-1121. https:// doi.org/10.1016/0009-2509(79)85017-4

Elnashaie, S. S. E. H. and Elshishini, S. S. (1996). Dynamic Modelling, Bifurcation and Chaotic Behaviour of Gas-Solid Catalytic Reactors. UK: Gordon and Breach Science Publishers.

Elnashaie, S. S. E. H. and Garhyan, P. (2003b). Conservation Equations and Modeling of Chemical and Biochemical Processes. New York: Marcel Dekker. https://doi.org/10.1201/9780203911501

Elnashaie, S. S. E. H. and Garhyan, P. (2004a). Chaotic Fermentation of Ethanol, United State Full Patent \#10/978,293 filed on 10/29/04a, August.

Elnashaie, S. S. E. H. and Grace, J. R. (2007). Complexity, bifurcation and chaos in natural and man-made lumped and distributed systems. Chemical Engineering Science, 62(13), 3295-3325. https://doi.org/10.1016/j.ces.2007.02.047

Elnashaie, S. S. E. H., Alhabdan, F. M. and Elshishini, S. S. (1993). The Vital Role of Mathematical Modeling in Chemical Engineering Education. Mathl. Comput. Modelling, 17(3), 3-11. https://doi.org/10.1016/0895$7177(93) 90034-\mathrm{V}$

Elnashaie, S. S. E. H., Chen, Z., Garhyan, P., Prasad, P. and Mahecha-Botero, A. (2006). Practical Implications of Bifurcation and Chaos in Chemical and Biological Reaction Engineering. International Journal of Chemical Reactor Engineering, 4, Review R1, 1-41. https:/ / doi.org/10.2202/1542-6580.1305

Elshishini, S. S. and Elnashaie, S. S. E. H. (1990). Digital simulation of industrial fluid catalytic cracking units, Bifurcation and its implications. Chem. Engng. Sci., 45, 553-559. https://doi.org/10.1016/0009-2509(90)87042Q

Fiksel, J. (2006). Sustainability and resilience: toward a systems approach. Science, Practice \& Policy, 2(2), 14-21. https://doi.org/10.1080/15487733.2006.11907980

Garhyan, P. and Elnashaie, S. S. E. H. (2004b). Static/Dynamic Bifurcation and Chaotic Behavior of an Ethanol Fermentor. Industrial and Engineering Chemistry Research (I\&ERC), 43(5), 1260-1273.

Garhyan, P. and Elnashaie, S. S. E. H. (2004c). Bifurcation Analysis of Two Continuous Membrane Fermentor Configurations for Ethanol Production. Chemical Engineering Science, 59(15), 3235-3268. https://doi.org/10.1016/j.ces.2004.05.003

Garhyan, P. and Elnashaie, S. S. E. H. (2004d). Bioethanol Production - Solving the Efficiency Bottleneck. The Chemical Engineer, 760, 30-32.

Grace, J. R. (2015). Fluidized bed catalytic reactors, Chapter 4 in Multiphase Catalytic Reactors: Theory, Design, Manufacturing and Applications, ed. Onsan ZI and Avci AK, Wiley, New York.

Hawking, S. W. (1989). A Brief History of Time, From the Big Bang to Black Holes, Bantam Press, London.

Iscol, L. (1970). The dynamics and stability of a fluid catalytic cracker. Joint Automatic Control Conference, Georgia, 602-607. 
Journal of Engineering Education (JEE). (2006). Special Report: The Research Agenda for the New Discipline of Engineering Education, 95, 259-261.

Journal of Engineering Education. (2005). JEE Strategic Plan, 2005-2010 Advancing Engineering Education Research Worldwide. Journal of Engineering Education, 94, 283-284.

Klein, J. T. (1990). Interdisciplinarity: History, Theory, and Practice. Detroit: Wayne State University Press, 1990.

Lattuca, L. R. (2001). Creating Interdisciplinarity: Interdisciplinary Research and Teaching among College and University Faculty: Vanderbilt University Press.

Lattuca, L. R., Voigt, L. J. and Fath, K. Q. (2004). Does Interdisciplinarity Promote Learning? Theoretical Support and Researchable Questions. The Review of Higher Education, 28, 23-48. https://doi.org/10.1353/rhe.2004.0028

Mahecha-Botero, A., Garhyan, P. and Elnashaie, S. S. E. H. (2004). Bifurcation and chaotic behaviour of a coupled acetylcholinesterase/choline acetyltransferase diffusion-reaction enzymes system. Chemical Engineering Science, 59 581-597. https://doi.org/10.1016/j.ces.2003.11.007

Meeting of the Finnish National Graduate School in Nanoscience (NGS-NANO) (2008). Helsinki University.

National Council for Science and the Environment (NCSE). (2003). A Report of the Third National Conference on Science, Policy and the Environment. Recommendations for Education for a Sustainable and Secure Future. Improving the scientific basis for environmental decision making, Washington, DC.

Ray, W. H. and C. M. (2000). Villa, Nonlinear dynamics found in polymerisation processes. Chem.Eng. Sci., 55, 275290. https:/ / doi.org/10.1016/S0009-2509(99)00323-1

Smith, J. M. (1974). Models in Ecology, Cambridge University Press, Cambridge.

Stewart, L. (1989). Does God Play Dice: The New Mathematics of Chaos, Penguin Books, London.

Teymour, F. (1989). The Dynamic Behavior of Free Radical Solution Polymerization Reactions in a Continuous Stirred Tank Reactor. PhD thesis, University of Wisconsin.

Teymour, F. and W. H. (1992a). Ray, The dynamic behaviour of continuous polymerization reactors - V. Experimental investigation of limit-cycle behavior for vinyl acetate polymerization. Chem.Eng. Sci., 47, 41214132. https:// doi.org/10.1016/0009-2509(92)85162-5

Teymour, F. and W. H. Ray (1992b). The dynamic behaviour of continuous polymerization reactors - VI. Complex dynamics in full-scale reactors. Chem. Eng. Sci., 47, 4133-4140. https://doi.org/10.1016/0009-2509(92)851636

Vigneault, A., Grace, J. R. and Elnashaie, S. S. E. H. (2012). Simulation of a compact multi-channel membrane reactor for the production of pure hydrogen via steam methane reforming. Chem. Eng. \& Technol, 35(8), 15201533. https://doi.org/10.1002/ceat.201200029

Vlachos, D. G., Vlachos, L., Schmidt, D. and Aris, R. (1991). Structures of Small Metal Clusters: Low Temperature Behavior, Army High Performance Computing Research Center Preprint, 91-70, University of Minnesota, Minneapolis.

Wei, J. (1988). Educating chemical engineers for the future, In Chemical Engineering in a Changing Environment, Engineering Foundation Conference, January 17-22, Santa Barbara, California, (Edited by S. L. Sandler and B. A. Finlayson), pp. 1-12. 